\title{
Detection of Network Attacks Based on Multiprocessing and Trace Back Methods
}

\author{
Vijayakumar $\mathrm{R}^{\mathrm{a}, 1}$, Vijay $\mathrm{K}^{\mathrm{b}}$, Sivaranjani $\mathrm{P}^{\mathrm{b}}$ and Priya $\mathrm{V}^{\mathrm{c}}$ \\ ${ }^{\mathrm{a}, \mathrm{b}}$ Assistant Professor, Department of CSE, Rajalakshmi Engineering College, \\ Chennai, India \\ ${ }^{\mathrm{c}}$ Assistant Professor, Department of CSE, PSNA College of Engg and Technology, \\ Dindigul, India
}

\begin{abstract}
The way of thinking of traffic observing for discovery of system assaults is predicated on a "gained information" viewpoint: current methods recognize either the notable assaults which they're customized to alarm on, or those strange occasions that veer off from a known typical activity profile. These philosophies depend on an expert structure which gives the ideal data, either with respect to "marks" of the striking attacks or as anomaly free traffic datasets, adequately rich to make delegate profiles for commonplace movement traffic. The theory talks about the limitations of current information-based system to recognize organize assaults in an inexorably unpredictable and advancing Web, Described by ever-rising applications and an ever-expanding number of most recent system assaults. In an oppositely inverse viewpoint, we place the weight on the occasion of solo recognition strategies, fit for distinguishing obscure system assaults during a unique situation with none past information, neither on the attributes of the assault nor on the gauge traffic conduct. In view of the perception that an outsized portion of system assaults are contained during a little division of traffic flows, the proposition exhibits an approach to join basic bunching strategies to precisely distinguish and portray malignant flows. to bring up the practicality of such an information autonomous methodology, a solid multi-bunching-based location technique is created and assess its capacity to recognize and portray arrange assaults with none past information, utilizing bundle follows from two genuine operational systems. The methodology is acclimated identify and describe obscure vindictive flows, and spotlights on the identification and portrayal of ordinary and notable assaults, which encourages the translation of results. When contrasted with the predominant DDoS traceback techniques, the proposed system has assortment of favorable circumstances - it is memory no concentrated, proficiently adaptable, vigorous against parcel contamination, and free of assault traffic designs. The consequences of inside and out test and reenactment considers are introduced to exhibit the adequacy and effectiveness of the proposed strategy. It's an uncommon test to traceback the wellspring of Circulated Disavowal of-Administration (DDoS) assaults inside the Web. In DDoS assaults, aggressors create a lot of solicitations to casualties through undermined PCs (zombies), with the point of keeping ordinary help or debasing from getting the norm of administrations. Because of this fundamental change, the proposed system conquers the acquired downsides of parcel stamping strategies, similar to weakness to bundle contaminations. The execution of the proposed strategy welcomes no changes on
\end{abstract}

${ }^{1}$ Vijayakumar R, Assistant Professor, Department of CSE Rajalakshmi Engineering College, Chennai, India.vijay.k@rajalakshmi.edu.in 
current steering programming. Moreover, this work builds up a hypothetical structure for assessing the insurance of IDS against mimicry assaults. It shows an approach to break the wellbeing of 1 distributed IDS with these strategies, and it tentatively affirms the capacity of various assaults by giving a worked model. The Project is intended by using Java 1.6 as face and MS SQL Server 2000 as backside. The IDE used is Net Beans 6.8.

Keywords: attacks; DDos; Dos; Trackback;

\section{Introduction}

System traffic checking has become a fundamental method for recognition of system assaults in the present Web. The chief test in recognizing system assaults is that these are a moving objective. It is beyond the realm of imagination to expect to know the various assaults that an aggressor may dispatch, in light of the fact that new assaults just as new variations of definitely realized assaults are consistently rising. Without a doubt, assaults have become both progressively various and refined throughout the long term. Two distinct methodologies are by a wide margin prevailing in ebb and flow research network and business recognition frameworks: signature-based location and peculiarity discovery. Regardless of being inverse in nature, the two methodologies share a typical drawback: they depend on the information gave by a specialist framework, generally a human master, to carry out the responsibility. We will hence allude to them as information-based identification draws near. From one viewpoint, signature-put together discovery frameworks are based with respect to a broad information on the specific attributes of each assault, alluded to as its "signature". Such frameworks are exceptionally successful to identify those notable assaults which they are customized to caution on. Regardless, they can't watch the framework against new attacks, just considering the way that they can't see what they haven't the foggiest. Likewise, assembling new marks includes manual review by human specialists, which isn't without a doubt, extravagant and inclined to blunders, yet additionally presents a significant inertness between the revelation of another assault and the development of its mark. In a system situation where, new assaults are continually showing up, such a manual cycle forces a genuine bottleneck on the guard abilities of the system. Then again, irregularity recognition depends on the presence of ordinary activity traffic examples to fabricate a pattern profile, recognizing inconsistencies as traffic exercises that stray from it. Such a methodology grants to distinguish new sorts of system assaults not seen previously, in light of the fact that these will normally go amiss from the developed standard.

Persuaded by the restrictions of information-based methodologies, another examination zone has developed in the most recent years, in light of an oppositely inverse way of thinking for location of bizarre traffic occasions: Unaided Oddity Discovery. Current bunch examination procedures: the absence of heartiness.

\section{Literature Survey}

DDoS attacks are engaged at crippling the setback's benefits, for instance, organize move speed, preparing force, and working system data structures. To dispatch a DDoS 
ambush, the attacker(s) first develops an arrangement of PCs that will be used to make the tremendous volume of traffic expected to decline any help to genuine customers of the individual being referred to. To cause this attack to orchestrate, aggressors find frail hosts on the framework. Frail hosts data on attack can be appointed follows: allowance subject to fragmentary information [5], certifiable framework mimicking [6] or reenactments [7], and veritable ambush and security between two organize assessments packs.

Plainly chasing down the aggressors (zombies), and further to the developers, is basic in understanding the attack challenge. The rundown of the current DDoS traceback strategies can be found in [3] and [4]. With everything taken into account, the traceback approaches rely upon package checking.

Pack checking procedures join the PPM and the DPM. The PPM framework endeavors to check groups with the switch's IP address information by probability on the close by switch, and the setback can reproduce the manners in which that the ambush bundles experienced. The PPM procedure is powerless against aggressors, as raised in [1], as attackers can send personification stepping information to the setback to bamboozle the individual being referred to. The precision of PPM is another issue in light of the fact that the stepped messages by the switches who are closer to the leaves (which suggests far away from the individual being referred to) could be overwritten by the downstream switches on the attack tree [2].

\section{System Assaults}

Despite the fact that we guarantee that our methodology can be utilized to identify and describe obscure malevolent streams, we center on the location and portrayal of standard and notable assaults, which encourages the understanding of results. Notwithstanding, we will accept no past information about these assaults, and in this manner treat them as totally obscure. Refusal of Administration (DoS), organize examines, Circulated DoS (DDoS), and worms' engendering are instances of standard assaults that day by day compromise the respectability and ordinary activity of the system.

\subsection{DDoS/DoS}

A DDoS/DoS assault is an endeavor to make a system asset (a specific help, arrange data transmission, and so on.) inaccessible to its expected (real) clients. In its most broad structure, a DoS/DDoS assault holds onto assets by utilizing or mentioning beyond what the casualty can deal with, keeping it from reacting to authentic solicitations.

\subsection{Worms spread}

A worm [8] is a malevolent self-duplicating program that utilizes the system to send duplicates of itself, contaminating different machines by misusing explicit weaknesses. A worm is typically used to introduce a secondary passage in the tainted PC, permitting the production of a "zombie" machine heavily influenced by the assailant. Systems of such machines are alluded to as "botnets", and are commonly used to dispatch enormous DDoS assaults. 
A system check [9] is a testing endeavor to distinguish the accessibility of a particular help on a wide range of machines. Distinguishing framework inspects is basic considering the way that such a development is commonly a predecessor of the expansion of a worm, and along these lines the harbinger of possible DDoS attacks. Framework checks are depicted by a lone source sending traffic to various complaints.

\subsection{Sub-Space Grouping and Proof Gathering}

The solo identification and portrayal calculation starts in the subsequent stage, utilizing as info the arrangement of streams caught in the abnormal space. An inconsistency is commonly recognized in various accumulation levels, and there are numerous heuristics to choose a specific conglomeration to use in the solo stage; for straightforwardness we will skirt this issue, and utilize any of the total levels where the irregularity was distinguished. Without loss of consensus, let $\mathrm{Y}=\{\mathrm{y} 1, \ldots, \mathrm{yn}\}$ be the arrangement of $\mathrm{n}$ streams in the hailed opening. Each stream yi to $\mathrm{Y}$ is portrayed by a lot of $\mathrm{m}$ traffic qualities or highlights. The rundown incorporates standard and essential traffic qualities, which grants to portray the distinguished assaults in simple to-decipher terms portrayal results.

Let $\mathrm{xi}=(\mathrm{xi}(1), \ldots, \mathrm{xi}(\mathrm{m})) 2 \mathrm{Rm}$ be the comparing vector of $\mathrm{m}$ traffic highlights portraying stream yi, and $\mathrm{X}=\{\mathrm{x} 1, \ldots, \mathrm{xn}\}$ the total framework of highlights, alluded to as the component space.

\section{Results and Discussions}

We assess the capacity of the solo calculation to recognize and to build a mark for various assaults in genuine rush hour gridlock follows from the open traffic vault of the WIDE undertaking. The WIDE operational system gives interconnection between various exploration foundations in Japan, just as association with various ISPs and colleges in the U.S..

\subsection{Detecting Network Scan}

Distinguishing a system filter initially recognize and portray a dispersed SYN organize examine coordinated to numerous casualty has under the equivalent/16 objective system. Parcels in Y are amassed in IPdst/24 streams, in this way we will distinguish the assault as a little size bunch.

The length of each opening is $\mathrm{T}=20$ seconds. As we clarified in segment III-A, the SSC-EA-based bunching calculation builds another comparability measure between streams in $\mathrm{Y}$. We will communicate this new similitude measure as a $\mathrm{n} \times \mathrm{n}$ network $\mathrm{S}$, in which component $\mathrm{S}(\mathrm{x}, \mathrm{y})$ speaks to the level of closeness between streams $\mathrm{x}$ and $\mathrm{y}$.

\subsection{Detecting a Distributed DoS attack}

Figure 1.(a,b) delineate various standards acquired in the identification of a SYN DDoS assault. Traffic is currently accumulated in Ipsrc/32 streams, and the assault is 
identified as a little size group. The examination of between streams likeness w.r.t. S chooses a minimal separated bunch, comparing to the arrangement of assaulting has. The acquired mark can be communicated as $(\mathrm{nDsts}==1)^{\wedge}(\mathrm{nSYN} / \mathrm{nPkts}>3)^{\wedge}$ $(\mathrm{nPkts} / \mathrm{sec}>4)$, which joined with the huge number of distinguished sources (nSrcs $>$ 5) affirms the idea of a SYN DdoS assault.

\subsection{Detecting outliers attacks}

On account of exception's recognition, the comparability measure gave by the SSC-EAbased calculation doesn't speak to between streams closeness; rather, it relates to the combined detachment of an anomaly to the greatest bunch in the distinctive sub-spaces. Let us first present the location of a SYN organize filter and an ICMP flooding assault utilizing the SSC-EA-based exception's recognition approach. Traffic is totaled in IPsrc/32 streams. Figure 1. shows the arranged divergence esteems acquired for the various streams, alongside their relating grouping.

The initial two most inaccessible streams compare to a profoundly appropriated SYN organize examine (in excess of 500 objective hosts) and an ICMP mock flooding assault coordinated to few casualties (ICMP divert traffic, coordinated towards port 0 ).

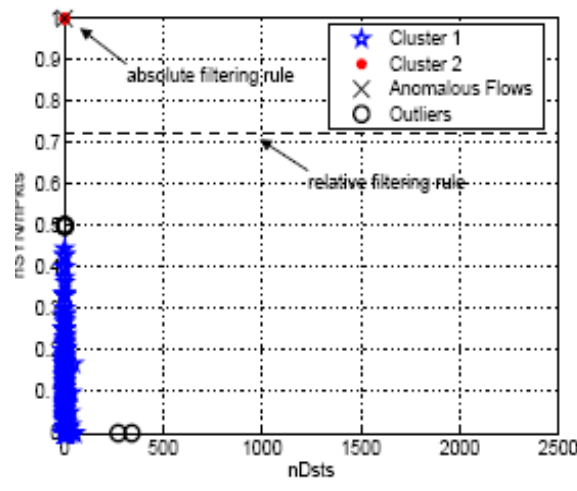

(a) $\mathrm{SYN} \operatorname{DDoS}(1 / 2)$

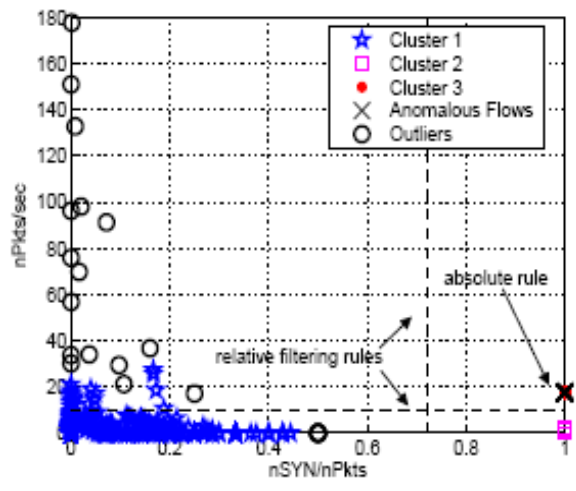

(b) $\mathrm{SYN}$ DDoS $(2 / 2)$

Figure 1 - SYN DDoS

\section{Conclusion and Future Scope}

In this work I question the capacity and stress the restrictions of current informationbased methodologies for identification of system assaults, especially with regards to an undeniably intricate and ever-advancing Web. In an oppositely inverse viewpoint, I place the accentuation on the improvement of unaided, information autonomous discovery calculations, which I accept is the following common advance in organize traffic observing for arrange security. As a proof-of-idea of how such a recognition approach could be really executed in the training, I have delineated a strong multigrouping-based identification technique and assessed its capacity to identify and portray standard system assaults with no past information, utilizing bundle follows from two genuine operational systems. 


\section{References}

[1] Wang.H, Jin.C and Shin.K.G, Defense against Spoofed IP Traffic Using Hop-Count Filtering, IEEE/ACM Trans. Networking, vol. 15, no. 1, pp. 40-53, Feb. 2007.

[2] K. Lu et al., Robust and Efficient Detection of DDoS Attacks for Large-Scale Internet, Computer Networks, vol. 51, no. 9, pp. 50365056, 2007.

[3] Kompella.R.R, Singh.S, and Varghese.G. On Scalable Attack Detection in the Network, IEEE/ACM Trans. Networking, vol. 15 , no. 1, pp. 14-25, Feb. 2007.

[4] Ayres.P.E et al., ALPi: A DDoS Defense System for High-Speed Networks, IEEE J. Selected Areas Comm., vol. 24, no. 10, pp. 18641876, Oct. 2006.

[5] R. Chen, J. Park, and R. Marchany, A Divide-and- Conquer Strategy for Thwarting Distributed Denialof- Service Attacks, IEEE Trans. Parallel and Distributed Systems, vol. 18, no. 5, pp. 577588, May 2007.

[6] A. Yaar, A. Perrig, and D. Song, StackPi: New Packet Marking and Filtering Mechanisms for DDoS and IP Spoofing Defense, IEEE J. Selected Areas Comm., vol. 24, no. 10, pp. 1853-1863, Oct. 2006.

[7] R. Vijayakumar, K. Selvakumar, K. Kulothungan and A. Kannan, Prevention of multiple spoofing attacks with dynamic MAC address allocation for wireless networks , 2014 International Conference on Communication and Signal Processing, Melmaruvathur, 2014, pp. 1635-1639, doi: 10.1109/ICCSP.2014.6950125.

[8] S. Fei, L. Zhaowen and M. Yan, A survey of internet worm propagation models, 2009 2nd IEEE International Conference on Broadband Network \& Multimedia Technology, Beijing, 2009, pp. 453457, doi: 10.1109/ICBNMT.2009.5348534.

[9] Hong.Q, T. Jianwei, Y. Ying, T. Zheng, Z. Hongyu and L. Shu, A Network Scanning Detection Method Based on TCP Flow State, 2018 3rd International Conference on Smart City and Systems Engineering (ICSCSE), Xiamen, China, 2018, pp. 419-422, doi: 10.1109/ICSCSE.2018.00089.

[10] Ambeth Kumar.V.D et.al.,.Performance Improvement Using an Automation System for Segmentation of Multiple Parametric Features Based on Human Footprint.for the Journal of Electrical Engineering \& Technology , vol. 10, no. 4, pp.1815-1821 , 2015. [http://dx.doi.org/10.5370/JEET.2015.10.4.1815]

[11] Ambeth Kumar.V.D et.al. A Survey on Face Recognition in Video Surveillance. Lecturer Notes on Computational and Mechanism, Vol. 30, pp: 699-708, 2019

[12] Ambeth Kumar.V.D .Precautionary measures for accidents due to mobile phone using IOT. Clinical eHealth, Volume 1, Issue 1, March 2018, Pages 30-35.

[13] Ambeth Kumar.V.D et,al, Enhancement in Footprint Image using Diverse Filtering Technique" Procedia Engineering journal, Volume 8, No.12, 1072-1080, 2012. [doi:10.1016/j.proeng.2012.01.965]

[14] Nanagasabapathy.K et.al. Validation system using smartphone luminescence.IEEE International Conference on Intelligent Computing, Instrumentation and Control Technologies (ICICICT), Pages: 235 - 239, 6-7 July 2017, Kannur, India

[15] B. Aravindh et.al, A novel graphical authentication system for secure banking systems.IEEE International Conference on Smart Technologies and Management for Computing, Communication, Controls, Energy and Materials, Pages: 177 - 183, 2-4 Aug. 2017, Vel Tech University, Chennai, India

[16] S.V. Ruphitha et.al. Management of Major Postpartum Haemorrhage by using Zigbee protocol - A Review .2021 6th International Conference on Inventive Computation Technologies (ICICT) (DOI: 10.1109/ICICT50816.2021.9358757)

[17] Indhumathi.M et.al .Healthcare Management of Major Cardiovascular Disease-A review. 2021 6th International Conference on Inventive Computation Technologies (ICICT), (DOI: 10.1109/ICICT50816.2021.9358519)

[18] Ambeth Kumar.V.D et.al. Cloud enabled media streaming using Amazon Web Services. IEEE International Conference on Smart Technologies and Management for Computing, Communication, Controls, Energy and Materials, Pages: 195-198, 2-4 Aug. 2017, India (DOI: 10.1109/icstm.2017.8089150)

[19] Ambeth Kumar.V.D et.al, (2016).An Efficient Security System for Data base Management from Illegal Access, IEEE International Conference on Wireless Communications, Signal Processing and Networking (WiSPNET), SSN Engineering College, Chennai, India, 23-25 March, 2016 